\title{
Plasma catestatin level predicts sPESI score and mortality in acute pulmonary embolism
}

Servet Izci ${ }^{1}$, Emrah Acar ${ }^{1}$, Mehmet Inanir ${ }^{2}$

${ }^{1}$ Department of Cardiology, Kartal Kosuyolu Kalp Ve Damar Hastanesi, Istanbul, Turkey ${ }^{2}$ Department of Cardiology, Bolu Abant Izzet Baysal University, Bolu, Turkey

Submitted: 23 December 2019

Accepted: 18 April 2020

Arch Med Sci Atheroscler Dis 2020; 5: e49-e56

DOI: https://doi.org/10.5114/amsad.2020.95562

Copyright $\odot 2020$ Termedia \& Banach

\author{
Corresponding author: \\ Dr. Emrah Acar \\ Department of Cardiology \\ Kartal Kosuyolu Kalp \\ Ve Damar Hastanesi ???? \\ Istanbul, Turkey \\ Phone: +905358819708 \\ E-mail: dreacar44@hotmail. \\ com
}

\begin{abstract}
Introduction: Acute pulmonary embolism (APE) is an emergent cardiothoracic disorder. The PESI score is used to estimate 30-day mortality in patients diagnosed with non-high-risk APE. Also, there are biomarkers for predicting prognosis and mortality in APE. Catestatin (CST) is accepted as a marker ofsympathetic nervous system activity which has been shown that the sympathetic nervous system activation can contribute pathogenesis in APE. So, we attempt herein to investigate the correlation of PE diagnosis and prognostic determination with plasma CST levels in PE patients.

Material and methods: Blood samples were drawn at admission for laboratory assays and CST measurements. Plasma levels of CST were measured by ELISA according to the manufacturer's instruction. Transthoracic echocardiography was performed for the assessment of RV dysfunction using a Toshiba Applio 500 echocardiographic system within $24 \mathrm{~h}$ of the admission.

Results: Plasma CST levels were higher in patients with APE than in the control group $(17.5 \pm 6.1 \mathrm{ng} / \mathrm{ml}$ vs. $27.3 \pm 5.7 \mathrm{ng} / \mathrm{ml}, p<0.001)$. Plasma CST levels were higher in the SPESI $\geq 1(n=72)$ than in the patients with sPESI $<1(37.3 \pm 6.1$ vs. $24.2 \pm 5.3 \mathrm{ng} / \mathrm{ml}, p<0.001)$. There was a positive correlation between CST level and SPESI score $( \pm 0.581, p<0.001)$. Mortality occurred in 20 patients with $\mathrm{SPESI} \geq 1(27.7 \%)$ and in 9 patients with $\mathrm{sPESI}$ $<1(10.2 \%)(p=0.010)$. Receiver operating characteristic (ROC) curve analysis using a cut-off level of $31.2 \mathrm{ng} / \mathrm{ml}$, and the CST level predicted mortality with a sensitivity of $100 \%$ and specificity of $52.6 \%$ (AUC $=0.883,95 \% \mathrm{Cl}$ : 0.689-0.921). Furthermore, the CST level was correlated with right ventricular dysfunction.
\end{abstract}

Conclusions: CST can predict SPESI score and mortality in patients with APE.

Key words: biomarker, catecholamine, catestatin, computed tomography, echocardiography, pulmonary embolism, sympathetic nervous system.

\section{Introduction}

Acute pulmonary embolism (APE) is an emergent cardiothoracic disorder, which can bring on serious complications such as haemodynamic instability, death, and chronic thromboembolic pulmonary hypertension [1]. One of the statistical data that best describes the seriousness of the problem is that APE causes nearly 200,000 deaths annually [1, 2]. Several risk graduation algorithms may be applied to delineate short-term mortality in patients with APE. The Pulmonary Embolism Severity Index (PESI) score is one of these algorithms and is utilised commonly for es- 
timating 30-day mortality in patients diagnosed with non-high risk (low or intermediate) APE [3]. The PESI score is calculated with variables related to vital signs as well as several clinical and haemodynamic measurements [3, 4]. Jimenez et al. made use of the PESI to build up the novel, simplified PESI (SPESI) [5]. They separated the patients with APE into two groups on the basis of SPESI score: SPESI $\geq 1$ for high SPESI and SPESI $<1$ for Iow SPESI. They reported that APE patients with a $S P E S I \geq 1$ had a higher mortality rate than the patients with SPESI < 1 (mortality rates: $8.9 \%$ vs. $1.1 \%)$. Various imaging techniques such as contrasted computed tomography (CT) scan of the chest, ventilation-perfusion (VQ) nuclear medicine imaging, and echocardiography (both transthoracic and transoesophageal), as well as laboratory parameters such as D-dimer, cardiac troponin, BNP, heart-type fatty acid-binding protein, and growth-differentiating factor-15 (GDF-15) are helpful in diagnosis and prognosis of acute PE [610]. It has been shown that the sympathetic nervous system can contribute to the pathogenesis of APE $[11,12]$. Catestatin (CST) is a residual, cationic, and hydrophobic peptide that is produced by proteolytic fragmentation of its forerunner structure chromogranin A (CHGA) [13]. CHGA is a protein located mainly in the secretory particles of chromaffin cells, postganglionic sympathetic neurons, and heart cells, where it is co-stacked and co-discharged with catecholamines [13-15]. CST is accepted as a part of the complex neurohumoral compensatory feedback system; higher levels of this peptide in peripheral blood might indirectly reflect increased neurohumoral burden [16]. For a long time it has been shown that CST can play a role in cardiovascular diseases such as hypertension, acute coronary syndrome, and cardiac remodelling [17-19], but there has been no study to investigate the role of CST in APE. Our hypothesis is that the plasma catestatin level in APE patients is helpful in the diagnosis of PE and is closely related to PE prognosis. In this study, we attempt to investigate the correlation of PE diagnosis and prognostic determination with plasma CST levels in PE patients upon hospitalisation.

\section{Material and methods}

We prospectively studied 160 consecutive patients (84 males, 76 females; median age: 61.9 years, range: 27-92 years) with diagnosed acute $\mathrm{PE}$, verified by contrasted computed tomography (CT) scan of the chest and/or ventilation-perfusion (VQ) nuclear medicine imaging, at Gumushane State Hospital and Kartal Kosuyolu Cardiovascular Research and Training Hospital. At admission clinical information was recorded and routine laboratory parameters were examined. Meanwhile, blood samples for CST were obtained and frozen at $-80^{\circ} \mathrm{C}$ until analysed.

Transthoracic echocardiography was performed for the evaluation of RV dysfunction using a Toshiba Applio 500 echocardiographic system within $24 \mathrm{~h}$ of admission, according to the guideline protocol from the British Society of Echocardiography [20]. Tricuspid annular plane systolic excursion (TAPSE) was obtained from an M-mode through the lateral tricuspid annular plane by measuring the amount of longitudinal motion of the annulus at peak systole. TAPSE $<1.7 \mathrm{~cm}$ indicated RV dysfunction [20]. RV fraction area change (FAC) was estimated as the ((RV end-diastolic area - end-systolic area)/end-diastolic area) $\times 100$. The RV endocardial border was traced in systole and diastole from the annulus, along the free wall, to the apex, and back along the interventricular septum using the apical four-chamber view. RV FAC < 35\% is associated with RV dysfunction [20]. To perform tissue doppler imaging (TDI)-derived Tricuspid Lateral Annular Systolic Velocity S' measurement, apical four-chamber windows were utilised with a tissue Doppler mode region of interest displaying the $\mathrm{RV}$ free wall. The pulsed Doppler sample volume was put on either the tricuspid annular line or the middle of the basal segment of the RV free wall. The velocity S' was stood for the highest systolic velocity. S' wave velocity $<9.5 \mathrm{~cm} / \mathrm{s}$ indicates RV dysfunction. For the calculation of pulmonary artery systolic pressure (PASP) a coaxial tricuspid regurgitation (TR) jet was defined in the parasternal long axis (RV inflow), parasternal short axis, or apical four-chamber view with colour Doppler. CW Doppler was used with a sweep speed of $100 \mathrm{~mm} / \mathrm{s}$ to obtain a convincing, non-feathered envelope. The peak velocity was then determined. Right atrial pressure was obtained by evaluating the size and distensibility of inferior vena cava during inspiration at rest and during forced inhalation, and this value was put in addition to the peak TR gradient. PASP > $40 \mathrm{~mm} \mathrm{Hg}$ is considered as RV dysfunction [20]. RV/LV basal diameter ratios were obtained offline from the parasternal short-axis two-dimensional view at the level of the papillary muscles with the RV free wall in view. RV and LV diameters were measured from the endocardial to endocardial surfaces at end-diastole. RV/LV ratios were calculated. A ratio of $>1$ measured at end-diastole suggests RV dysfunction [20].

The baseline features of the study patients were obtained from patients' recordings. Sex, age, heart rate, systemic blood pressure, troponin, D-dimer, and NT-proBNP were acquired from the statements of the medical contact examination implemented in the emergency room. According to the European Society of Cardiology (ESC) guidelines, 
PESI scores and SPESI scores of all of the patients were estimated by using the clinical data and the haemodynamic parameters [2, 3]. Patients were put into two groups on the basis of SPESI score: SPESI $<1(n=82)$ and $\mathrm{SPESI} \geq 1(n=78)$.

Venous peripheral blood samples were drawn at admission for routine laboratory assays and CST measurements. The serum samples for CST were placed into tubes containing potassium EDTA and immediately frozen at $-80^{\circ} \mathrm{C}$ until assayed. Plasma levels of CST were estimated by enzyme-linked immunosorbent assay (ELISA) according to the manufacturer's instruction (Cat. \# EK-053-29, ELISA kit, Phoenix Pharmaceutical Inc., Burlingame, CA). The minimum detection level for CST was $0.06 \mathrm{ng} / \mathrm{ml}$. CST plasma levels were also measured in the control group, which included 97 subjects (42 males, 55 females; median age: 57.4 years, range: $21-84$ years) in good general condition without PE or other significant cardiovascular comorbidities. These measurements were conducted by an investigator blinded to the origins of the samples.

\section{Statistical analysis}

The statistical analyses were conducted utilising IBM SPSS Statistics for Windows, Version 20.0 (IBM Corp., Armonk, USA). SPESI score < 1 was the criterion used to set the two study groups. Continuous variables were presented as mean \pm SD or median, and data were presented as a percentage for categorical variables. The Kolmogorov-Smirnov test was employed to test the distribution pattern. Categorical variables were tested with Pearson's $\chi^{2}$ test. Normally distributed continuous variables were examined with an independent $t$-test, and those with non-normal distribution were examined using the Mann-Whitney $U$ test. Pearson's correlation analysis was applied to check correlations between variables. In all analyses, statistical significance was defined as $p<0.05$.

\section{Result}

A total of 160 patients who were diagnosed with APE using pulmonary computed tomography angiography or ventilation/perfusion scintigraphy were included in the research. The baseline characteristics of the study patients are shown in Table I. The first group included 72 patients with SPESI $\geq 1$, and the second group comprised 88 patients with $\mathrm{SPESI}<1$. There were no significant differences between the groups in age and gender distribution, or presence of diabetes mellitus, hypertension, smoking, and history of coronary artery disease (Table I). Heart rate was significantly higher in patients with SPESI $\geq 1$ ( $p<0.001)$. Also, systemic blood pressure was different between the groups ( $p=0.027)$. In biomarker test results, there were no significant differences in troponin and D-dimer values. However, there was a difference between the groups in NT-proBNP level $(p<0.001)$. Biomarker results of the patients are shown in Table II. Median plasma CST level was higher in patients with APE than in the control group $(17.5 \pm 6.1 \mathrm{ng} / \mathrm{ml}$ vs. $27.3 \pm 5.7 \mathrm{ng} /$ $\mathrm{ml}, p<0.001$ ) (Figure 1). Median plasma CST level was higher in the SPESI $\geq 1$ patients $(n=$

Table I. The baseline characteristics of study patients

\begin{tabular}{|c|c|c|c|c|c|c|c|}
\hline \multirow[t]{2}{*}{ Variable } & \multicolumn{3}{|c|}{ sPESI $\geq 1(n=72)$} & \multicolumn{3}{|c|}{ sPESI $<1(n=88)$} & \multirow[t]{2}{*}{$P$-value } \\
\hline & $n$ & $\%$ & Mean \pm SD & $n$ & $\%$ & Mean \pm SD & \\
\hline Age [years] & & & $62.22 \pm 11.79$ & & & $60.57 \pm 12.27$ & 0.438 \\
\hline \multicolumn{8}{|l|}{ Sex: } \\
\hline Male & 31 & 42.9 & & 53 & 60.7 & & 0.015 \\
\hline Female & 41 & 57.1 & & 35 & 39.3 & & \\
\hline Smoking & 47 & 65.2 & & 50 & 57.1 & & 0.149 \\
\hline Hypertension & 29 & 40.2 & & 32 & 36.3 & & 0.492 \\
\hline Diabetes mellitus & 31 & 42.7 & & 33 & 37.5 & & 0.286 \\
\hline Coronary artery disease & 11 & 15.7 & & 15 & 16.9 & & 0.801 \\
\hline Systemic BP [mm Hg] & & & $109.91 \pm 17.42$ & & & $115.39 \pm 21.37$ & 0.027 \\
\hline Heart rate [bpm] & & & $116.45 \pm 22.37$ & & & $82.94 \pm 14.29$ & $<0.001$ \\
\hline PESI score & & & $93.27 \pm 19.91$ & & & $71.38 \pm 17.24$ & $<0.001$ \\
\hline $\mathrm{BMI}\left[\mathrm{m} / \mathrm{kg}^{2}\right]$ & & & $23.6 \pm 2.2$ & & & $23.4 \pm 2.1$ & 0.581 \\
\hline
\end{tabular}


Table II. Biomarker blood tests parameters of the patients

\begin{tabular}{|lccc|}
\hline Biomarker & sPESI $\geq 1(n=72)$ & sPESI $<1(n=88)$ & $P$-value \\
\hline D-dimer, median (min.-max.) $[\mathrm{ng} / \mathrm{ml}]$ & $1428(430-32490)$ & $2152(470-25550)$ & 0.142 \\
\hline Troponin, median (min.-max.) $[\mathrm{pg} / \mathrm{ml}]$ & $14.7(1.0-642.0)$ & $16.1(1.0-507.0)$ & 0.537 \\
\hline NT-proBNP, median (min.-max.) $[\mathrm{pg} / \mathrm{ml}]$ & $6041(2043-25000)$ & $772.8(16-29071)$ & $<0.001$ \\
\hline CST, mean \pm SD (min.-max.) $[\mathrm{ng} / \mathrm{ml}]$ & $37.3 \pm 6.1(0.4-99.6)$ & $24.2 \pm 5.3(0.1-69.3)$ & $<0.001$ \\
\hline
\end{tabular}

CST - catestatin, NT-proBNP - N-terminal pro brain natriuretic peptide, SPESI - Simplified Pulmonary Embolism Severity Index.

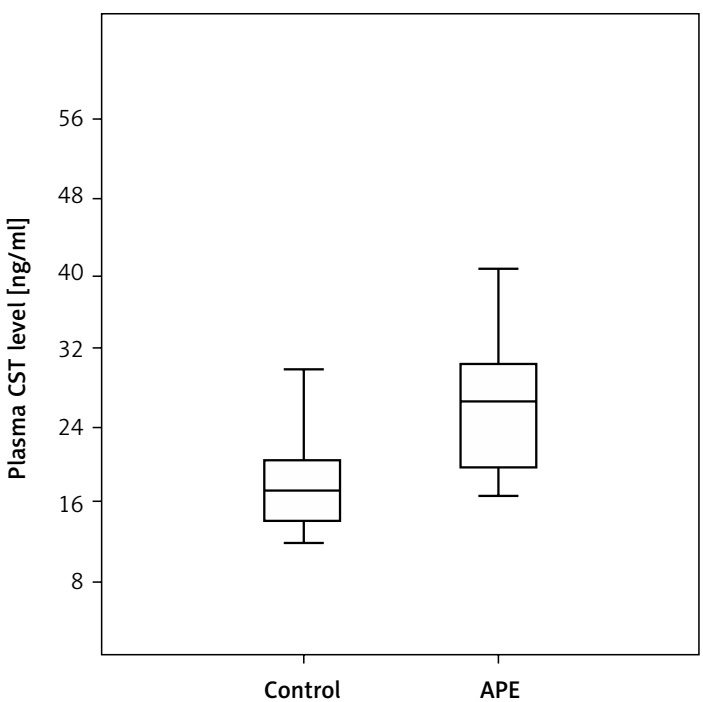

Figure 1. Plasma CST level in control group and in APE

72) than in the patients with SPESI $<1(37.3 \pm 6.1$ vs. $24.2 \pm 5.3 \mathrm{ng} / \mathrm{ml}, p<0.001$ ) (Figure 2). There was a positive correlation between CST level and sPESI score $(+0.581, p<0.001)$. Among study population, in-hospital mortality was observed in 29 patients. Mortality occurred in 20 patients

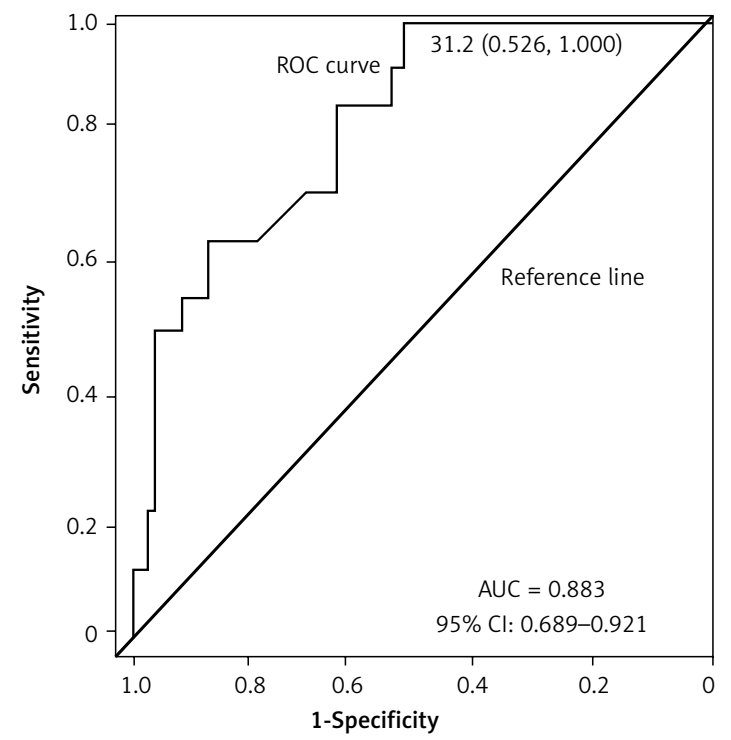

Figure 3. Receiver operating characteristic curve analysis

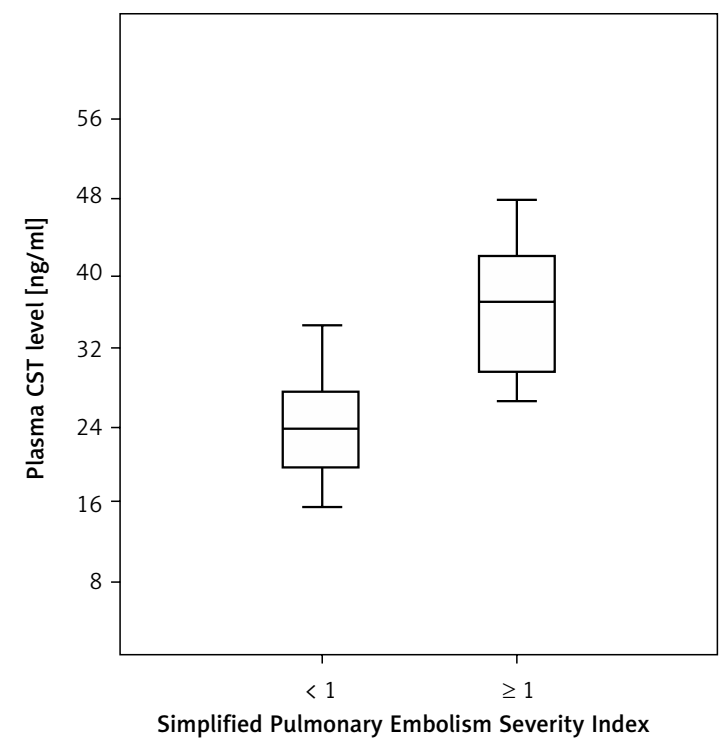

Figure 2. Plasma CST level according to the sPESI score

with SPESI $\geq 1(27.7 \%)$ and in nine patients with SPESI < $1(10.2 \%)(p=0.010)$. There was a positive correlation with the mortality rate and CST level; the mortality was higher in patients with higher CST level $(p=0.006)$. In receiver operating characteristic (ROC) curve analysis using a cut-off level of $31.2 \mathrm{ng} / \mathrm{ml}$, CST level predicted mortality with a sensitivity of $100 \%$ and specificity of $52.6 \%$ (ROC area under curve $=0.883 ; 95 \% \mathrm{Cl}$ : 0.689-0.921) (Figure 3).

The patients' echocardiographic findings are detailed in Table III. TAPSE was lower in the patients with SPESI $\geq 1$ than the SPESI $<1$ group $(16.91=2.51$ vs. $23.59+2.49, p<0.001)$. CST level was negatively correlated with TAPSE $(-0.256$, $p=0.004)$. In patients with SPESI $\geq 1$, S' was lower than the $\mathrm{sPESI}<1$ group $(8.36 \pm 2.06 \mathrm{~cm} / \mathrm{s}$ vs. $13.29 \pm 2.04 \mathrm{~cm} / \mathrm{s} ; p<0.001)$. FAC percentage was lower in the patients with $S P E S I \geq 1$ than in the patients with sPESI $<1(29.33 \pm 3.21$ vs. $40.29 \pm 3.34$, $p<0.001)$. S' value and FAC were inversely correlated with CST level $(-0.349, p<0.001$ and $-0.598, p<0.001$, respectively). Also, there was a difference in RV/LV basal diameter ratio between the $\mathrm{SPESI}<1$ group and the SPESI $\geq 1$ group (1.09 \pm 0.11 vs. $0.83 \pm 0.09, p<0.001)$. CST level was positively correlated with RV/LV basal diameter ratio 
Table III. Echocardiographic findings of the patients

\begin{tabular}{|lccc|}
\hline Variables & sPESI $\geq 1(n=72)$ & sPESI $<1(n=88)$ & $P$-value \\
\hline PASP $[\mathrm{mm} \mathrm{Hg}]$ & $41.8 \pm 10.24$ & $35.37 \pm 10.11$ & 0.008 \\
\hline TAPSE $[\mathrm{mm}]$ & $16.91 \pm 2.51$ & $23.59 \pm 2.49$ & $<0.001$ \\
\hline S' $[\mathrm{cm} / \mathrm{s}]$ & $8.36 \pm 2.0 .6$ & $13.29 \pm 2.04$ & $<0.001$ \\
\hline FAC $(\%)$ & $29.33 \pm 3.21$ & $40.29 \pm 3.34$ & $<0.001$ \\
\hline RV/LV basal diameter ratio & $1.09 \pm 0.11$ & $0.83 \pm 0.09$ & $<0.001$ \\
\hline
\end{tabular}

FAC - fractional area change, $L V$ - left ventricle, PASP - pulmonary artery systolic pressure, RV - right ventricle, S' - tissue doppler derived Right ventricle free Wall systolic velocity, TAPSE - tricuspid annular plane systolic excursion.

$(+0.324, p<0.001)$. There was no correlation with PASP and CST level.

\section{Discussion}

In the present study, plasma CST levels were higher in patients with APE than in the control group $(17.5 \pm 6.1 \mathrm{ng} / \mathrm{ml}$ vs. $27.3 \pm 5.7 \mathrm{ng} / \mathrm{ml}$ $p<0.001)$. Also, plasma CST levels wre higher in the $\mathrm{SPESI} \geq 1$ group $(n=72)$ than in the patients with $\mathrm{SPESI}<1(37.3 \pm 6.1$ vs. $24.2 \pm 5.3 \mathrm{ng} / \mathrm{ml}$, $p<0.001$ ) (Figure 2). There was a positive correlation between CST level and SPESI score $(+0.581$, $p<0.001)$. Furthermore, CST level was correlated with the RV dysfunctional parameters analysed in the study. There was a positive correlation with the mortality rate and CST level; the mortality was higher in patients with higher CST level ( $p=$ 0.006). In ROC curve analysis using a cut-off level of $31.2 \mathrm{ng} / \mathrm{ml}$, CST level predicted mortality with a sensitivity of $100 \%$ and specificity of $52.6 \%$ (ROC area under curve $=0.88395 \% \mathrm{Cl}: 0.689-0.921)$.

Acute pulmonary embolism is an emergent cardiothoracic disorder, which can bring on serious complications such as haemodynamic instability, death, and chronic thromboembolic pulmonary hypertension [1]. One of the statistical data that best describes the seriousness of the problem is that APE causes nearly 200,000 deaths annually $[1,2]$. Several risk graduation algorithms may be applied to delineate short-term mortality in patients with APE. The PESI score is one of these algorithms and is utilised commonly for estimating 30-day mortality in patients diagnosed with non-high-risk (low or intermediate) APE [3]. The PESI score is calculated with variables related to vital signs as well as several clinical and haemodynamic measurements [3, 4]. Jimenez et al. made use of the PESI to build up the novel, simplified PESI (sPESI) [8]. They separated the patients with APE into two groups on the basis of SPESI score: SPESI $\geq 1$ for high SPESI and $\mathrm{SPESI}<1$ for low SPESI. They reported that APE patients with a $S P E S I \geq 1$ had a higher mortality rate than the patients with sPESI < 1 (mortality rates: $8.9 \%$ vs. $1.1 \%)$. Various imaging techniques such as con- trasted computed tomography (CT) scan of the chest, ventilation-perfusion (VQ) nuclear medicine imaging, and echocardiography (both transthoracic and transoesophageal), as well as laboratory parameters such as D-dimer, cardiac troponin, BNP, heart-type fatty acid-binding protein, and growth-differentiating factor-15 (GDF-15) are helpful in diagnosis and prognosis of acute PE [6-10].

When the pulmonary arterial tree is plugged with thrombi, pulmonary blood flow is hindered. Locally releasing humoral mediators such as thromboxane A2, serotonin, and endothelins leads to pulmonary vasoconstriction [21]. It raises pulmonary vascular resistance. Right ventricle dilatation ensues, and its contractile properties are altered by the Frank-Starling mechanism [22]. Cardiac output is reduced and hypoxia occurs with ventilation perfusion mismatch. In total, these changes invoke sympathetic nervous system activation [23, 24]. A 'catecholamine rain' begins in the body with sympathetic activity. It has been shown that the sympathetic nervous system can take part in the pathogenesis of APE $[11,12]$. This compensatory response aggravates pulmonary vasoconstriction at the expense of their function to increase the reduced cardiac output [24]. Recently in a rabbit model of APE, it was shown that increased sympathetic activity in the whole lung tissue may aggravate the pulmonary spasm in APE, thus contributing to pulmonary hypertension, peripheral circulatory failure, and sympathetic activation in the setting of APE [25]. Additionally, increased levels of epinephrine, a catecholamine that is the main neurotransmitter of the sympathetic nervous system, lead to an APE-induced right ventricular myocarditis [26]. Hence, increased sympathetic activity fulfils a vicious cycle for right ventricular dysfunction via increasing RV afterload, aggravating pulmonary vasospasm, and triggering an epinephrine-induced RV myocarditis. CST is a residual, cationic, and hydrophobic peptide, which is produced by proteolytic fragmentation of its forerunner structure chromogranin A (CHGA) [13]. CHGA is a protein 
located mainly in the secretory particles of chromaffin cells, postganglionic sympathetic neurons, and heart cells, where it is co-stacked and co-discharged with catecholamines [13-15]. CST functions in several ways and acts strongly on nicotinic acetylcholine receptors on adrenal medullary chromaffin cells or adrenergic neurons to inhibit catecholamine secretion. Hence, it is a member of the negative feed-back system of the sympathetic nervous system $[13,14]$. Also, CST induces peritoneal and pleural mast cells involving inhibitory G-proteins (GTP-binding regulatory proteins) to stimulate release of the vasodilator histamine, and thus causes hypotension [13, 15]. In an unconventional way, CST has an impact on the central nicotinic-cholinergic synapses in the nucleus of the tractus solitarius in the baroreceptor control region of the brain stem to promote baroreceptor sensitivity, augment parasympathetic activity, and supress sympathetic activity [13]. These activities may give rise to the blood pressure- and heart rate-lowering functions of this endogenous peptide. Because CST is accepted as a part of the complex neurohumoral compensatory feedback system, higher levels of this peptide in peripheral blood might indirectly reflect increased neurohumoral burden [16]. However, it may be insufficiently compensatory when the level of CST increases in patients with APE. Therefore, it might not achieve balance between catecholamine and CST at a higher level, and this is still not enough to compensate for the damage to the body caused by sympathetic and catecholamine toxicity [18].

For a long time, it has been shown that CST can play a role in cardiovascular diseases. A lower plasma level of CST has been associated with development of hypertension [17]. In a study by Zhu et al. it was shown that plasma CST level and the prognosis of patients with acute myocardial infarction (AMI) is correlated [18]. They noticed that the CST level decreased on admission and increased with time in AMI. The CST level on day 3 was a good predictor of outcomes in patients with AMI, with good specificity as well as sensitivity. They explained that the increase of CST was the compensatory response of the sympathetic nervous system activity, which release catecholamine and harms the myocardium, so the CST level during the acute phase of AMI could reflect the degree of sympathetic system activation. Pei et al. revealed that the CST level can be helpful as a new biological marker for the prediction of malignant arrhythmia in patients with AMI [27]. They depicted that the incidence of malignant arrhythmia in patients with $\mathrm{AMI}$ increased significantly during hospitalisation as the CST level elevated. In another study, it was suggested that catestatin might be a novel marker reflecting LV remodelling in the myocardium following AMI [19]. Boravac et al. found that among patients with acutely decompensated heart failure, serum catestatin levels were higher [16]. They explained that higher CST levels are associated with beneficial metabolic and cardioprotective effects, but high CST levels reflect higher disease severity and probably parallel increased neurohumoral activity that might translate to adverse outcomes during the natural course of the disease.

However, until now there has been no study evaluating the possible linkage between APE, in which there is also an increased sympathetic nervous system activation, and CST level. In the present study, we found elevated CST level in patients with APE. There is an increased catecholaminergic milieu in the APE settings $[11,12]$. Hence it is plausible that CST levels might present this milieu. It has been shown that CST exhibited cardiosuppressive action and had an antagonistic impact on $\beta$-adrenergic positive inotropism and endothelin-1 mediated vasoconstriction, especially under highstress conditions [28-30]. Hence, CST may worsen some haemodynamic parameters in patients with APE. Meanwhile, it is possible that CST may play a protective role against overexaggerated catecholamine response [30, 31]. With the activation of the sympathetic nervous system, there is a rise in catecholamine release. In this earliest phase, CST levels are decreased and there is less inhibition of catecholamine liberation because CST plays a role in hindering catecholamine discharge from both chromaffin cells and neuro-adrenergic neurons through a reversible and non-competitive way. However, plasma CST levels change dynamically. In the later phase of the sympathetic system activation, compensatory catecholaminergic activation causes CST to increase and may reduce the harmful effects of increased catecholamine levels [31].

We detected that a high level of CST can predict mortality in APE. We speculate that a high CST level in APE might represent overall burden of the pathophysiology, and thus can predict the mortality and help in the risk stratification in APE.

There are several limitations to the study. First, the timing of blood sample collection may influence the measured levels of CST. We lack data on CST dynamics at various time-points, and no causal inferences could be made due to the possibility of interference of non-measured confounders. Second, CST levels were tested by an ELISA kit, for which there is a possibility that the assay detects the whole chromogranin A. According to the manufacturer's instructions, the ELISA kit utilised in this study did not have cross-reactivity with chromogranin A and vasostatin, and previously published studies have used this same assay. However, it would have been prudent to perform repeat testing on the samples using a kit specific for chromogranin A and to calculate any measure- 
ment error between the two assays. Third, testing of catecholamine levels may have strengthened the results; therefore, we could not quantify catecholamine burden or sympathetic "excess" in the study patients. Fourth, due to the lack of mechanistic insights, associations of CST with the measured parameters cannot be verified through a direct pathophysiological link, thereby requiring further elucidation in future mechanistic preclinical and translational studies.

In conclusion, the results of our study point to CST as a promising new biomarker for risk stratification of patients with acute PE. On the basis of these findings, we believe that the prognostic value of CST in this setting deserves further investigation. Prognostic algorithms using multimarker approaches, or the combination of CST with imaging procedures (echocardiography or computed tomography), need to be validated in larger numbers of patients with PE. From the diagnostic and therapeutic perspective, the critical question is whether CST, being a sensitive global indicator of poor outcome in acute pulmonary embolism, can assist in imaging studies or myocardium-specific biomarkers in identifying: (1) diagnostic assistance for patients with symptoms such as dyspnoea, chest pain and palpitation, or syncope; and (2) non-high-risk patients with APE, who may benefit from early thrombolytic or surgical treatment.

\section{Conflict of interest}

The authors declare no conflict of interest.

\section{References}

1. Heit JA. The epidemiology of venous thromboembolism in the community. Arterioscler Thromb Vasc Biol 2008; 28: 370-2.

2. Cohen AT, Agnelli G, Anderson FA, et al. Venous thromboembolism (VTE) in Europe. The number of VTE events and associated morbidity and mortality. Thromb Haemost 2007; 98: 756-64.

3. Chan CM, Woods C, Shorr AF. The validation and reproducibility of the pulmonary embolism severity index. J Thromb Haemost 2010; 8: 1509-14.

4. Aujesky D, Roy PM, Verschuren F, et al. Outpatient versus inpatient treatment for patients with acute pulmonary embolism: an international, open-label, randomised, non-inferiority trial. Lancet 2011; 378: 41-8.

5. Jiménez D, Aujesky D, Moores L, et al. RIETE Investigators. Simplifcation of the pulmonary embolism severity index for prognostication in patients with acute symptomatic pulmonary embolism. Arch Intern Med 2010; 170: 1383-9.

6. Aujesky D, Roy PM, Guy M, et al. Prognostic value of D-dimer in patients with pulmonary embolism. Thromb Haemost 2006; 96: 478-82.

7. Becattini C, Vedovati M.C, Agnelli G. Prognostic value of troponins in acute pulmonary embolism: a meta-analysis. Circulation 2007; 116: 427-33.
8. Coutance G, Le Page O, Lo T, Hamon M. Prognostic value of brain natriuretic peptide in acute pulmonary embolism. Crit Care 2008; 12: R109.

9. Bajaj A, Rathor P, Sehgal V, et al. Risk stratification in acute pulmonary embolism with heart-type fatty acidbinding protein: a meta-analysis. J Crit Care 2015; 30: 1151.e1-7.

10. Duran L, Kayhan S, Guzel A, et al. The prognostic values of GDF-15 in comparison with NT-proBNP in patients with normotensive acute pulmonary embolism. Clin Lab 2014; 60: 1365-71.

11. Faxon $\mathrm{HH}$, Anderson RM. Stellate block as an adjunct to the treatment of pulmonary embolism. N Engl J Med 1951; 244: 586-90.

12. Szold O, Khoury W, Biderman P, Klausner JM, Halpern P, Weinbroum AA. Inhaled nitric oxide improves pulmonary functions following massive pulmonary embolism: a report of four patients and review of the literature. Lung 2006; 184: 1-5.

13. Mahapatra NR. Catestatin is a novel endogenous peptide that regulates cardiac function and blood pressure. Cardiovasc Res 2008; 80: 330-8.

14. Helle KB, Corti A, Metz-Boutigue MH, Tota B. The endocrine role for chromogranin $A$ : a prohormone for peptides with regulatory properties. Cell Mol Life Sci 2007; 64: 2863-86.

15. Pieroni M, Corti A, Tota B, et al. Myocardial production of chromogranin $A$ in human heart: a new regulatory peptide of cardiac function. Eur Heart J 2007; 28: 1117-27.

16. Borovac JA, Glavas D, Susilovic Grabovac Z, Supe Domic D, D'Amario D, Bozic J. Catestatin in acutely decompensated heart failure patients: insights from the CATSTAT-HF Study. J Clin Med 2019; 8. pii: E1132.

17. O'Connor DT, Kailasam MT, Kennedy BP, Ziegler MG, Yanaihara N, Parmer RJ. Early decline in the catecholamine release-inhibitory peptide catestatin in humans at genetic risk of hypertension. J Hypertens 2002; 20: 1335-45.

18. Zhu D, Xie H, Wang X, Liang Y, Yu H, Gao W. Correlation of plasma catestatin level and the prognosis of patients with acute myocardial infarction. PLoS One 2015; 10: e0122993.

19. Zhu D, Xie H, Wang X, Liang Y, Yu H, Gao W. Catestatin - a novel predictor of left ventricular remodeling after acute myocardial infarction. Sci Rep 2017; 7: 44168.

20. Augustine DX, Coates-Bradshaw LD, Willis J, Harkness A, Ring L, Grapsa J. Echocardiographic assessment of pulmonary hypertension: a guideline protocol from the British Society of Echocardiography. Echo Res Pract 2018; 5: G11-24.

21. Smulders YM. Pathophysiology and treatment of haemodynamic instability in acute pulmonary embolism: the pivotal role of pulmonary vasoconstriction. Cardiovasc Res 2000; 48: 23-33.

22. Greyson CR. Pathophysiology of right ventricular failure. Crit Care Med 2008; 36 (1 Suppl): S57-65.

23. Tverskaia MS, Makarova LD, Sergeeva NA, Virganskiī AO, Savvina $S$. The neurohormonal changes in acute pulmonary embolism (clinico experimental research). Biull Eksp Biol Med 1993; 116: 29-31.

24. de Man FS, Handoko ML, Guignabert C, Bogaard HJ, Vonk-Noordegraaf A. Neurohormonal axis in patients with pulmonary arterial hypertension: friend or foe? Am J Respir Crit Care Med 2013; 187: 14-9.

25. Wang Y, Yu D, Yu Y, et al. Potential role of sympathetic activity on the pathogenesis of massive pulmonary embolism with circulatory shock in rabbits. Respir Res 2019; 20: 97. 
26. Begieneman MP, van de Goot FR, van der Bilt IA, et al. Pulmonary embolism causes endomyocarditis in the human heart. Heart 2008; 94: 450-6.

27. Pei Z, Ma D, Ji L, et al. Usefulness of catestatin to predict malignant arrhythmia in patients with acute myocardial infarction. Peptides 2014; 55: 131-5.

28. Angelone T, Quintieri AM, Brar BK, et al. The antihypertensive chromogranin a peptide catestatin acts as a novel endocrine/paracrine modulator of cardiac inotropism and lusitropism. Endocrinology 2008; 149: 4780-93.

29. Imbrogno S, Garofalo F, Cerra MC, Mahata SK, Tota B. The catecholamine release-inhibitory peptide catestatin (chromogranin A344-363) modulates myocardial function in fish. J Exp Biol 2010; 213: 3636-43.

30. Mazza R, Gattuso A, Mannarino C, et al. Catestatin (chromogranin A344-364) is a novel cardiosuppressive agent: inhibition of isoproterenol and endothelin signaling in the frog heart. Am J Physiol Heart Circ Physio 2008; 295: H113-22.

31. Petersen CL, Nielsen JR, Petersen BL, Kjaer A. Catecholaminergic activation in acute myocardial infarction: time course and relation to left ventricular performance. Cardiology 2003; 100: 23-8. 